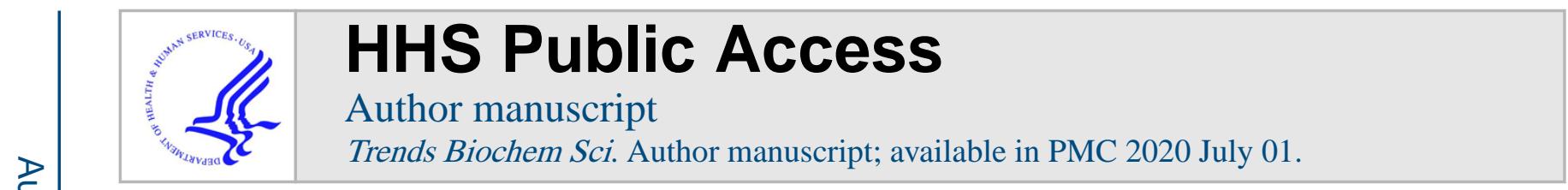

Published in final edited form as:

Trends Biochem Sci. 2019 July ; 44(7): 640-641. doi:10.1016/j.tibs.2019.03.006.

\title{
Getting a Grip on Greasy Molecules
}

\section{Timothy W. Bumpus ${ }^{1}$ Jeremy M. Baskin ${ }^{1,}$, \\ ${ }^{1}$ Department of Chemistry and Chemical Biology and Weill Institute for Cell and Molecular Biology, Cornell University, Ithaca, NY 14853, USA}

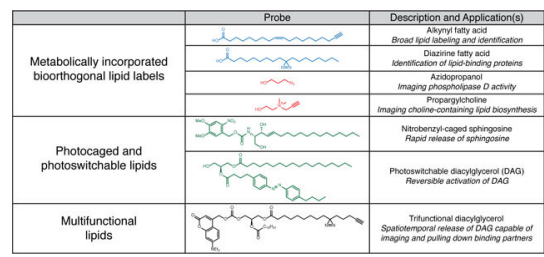

Lipids are structurally and functionally diverse biological metabolites that have long proven difficult to study, particularly as targets for molecular imaging. This challenge arises from their small size, hydrophobicity, rapid diffusion and trafficking rates, and, perhaps most critically, their status as metabolites whose structures and biosynthesis are not directly encoded in the genome, making them inaccessible to direct fusion with fluorescent proteins.

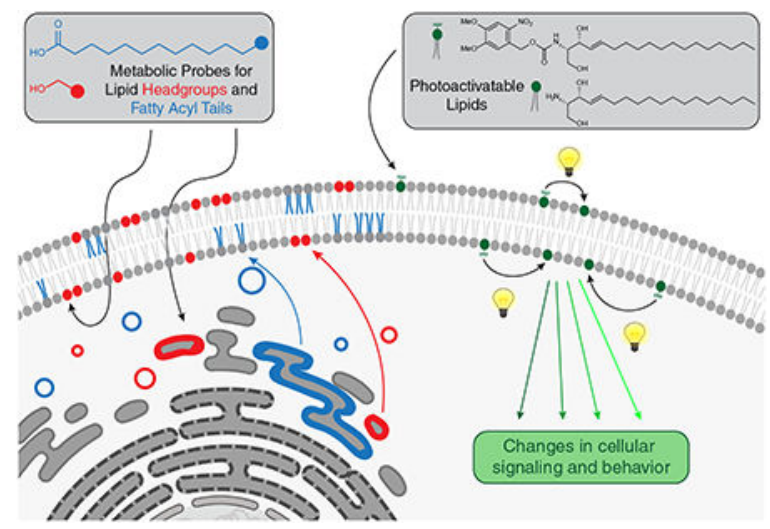

As a result of these challenges, most analytical methods for lipids rely on measuring lipid levels after sample homogenization and lipid extraction. These studies are critical to our understanding of lipid biology, but bulk biochemical, ex vivo analysis carries no spatial information and thus obscures the role of localization in downstream biological outcomes. Recent advances have focused on new approaches to study lipids within intact cells and tissues to better elucidate the spatial component of the function of these critical molecules.

“Correspondence: jeremy.baskin@ cornell.edu (J.M. Baskin). 


\section{ADVANTAGES:}

New analytical tools in lipid biology, such as metabolic labeling followed by bioorthogonal ligation, enable real-time observation of lipid signaling in living cells and link the spatial and temporal domains of signaling.

Photoactivatable lipids enable rapid release of signaling lipids with precise control of time and localization for highly targeted studies of signaling events in live cells.

Multifunctional lipids, which can be tailored to specific applications, couple the spatiotemporal precision of photoactivatable groups with photo-crosslinkers and bioorthogonal handles to enable visualization or enrichment of lipids and their interaction partners.

\section{CHALLENGES:}

Metabolic labeling of lipids relies on native biosynthetic enzymes to incorporate unnatural groups. Tagged lipids are produced in the same membrane environment as their natural counterparts, which is desirable. Yet, because of their different chemical makeups, these lipids may be trafficked differently.

Different lipids can share biosynthetic enzymes, limiting the points at which metabolic probes may be introduced to maintain selectivity to a desired lipid.

When certain highly functionalized, exogenous lipid analogs are added to cells, they may be trafficked differently and incorporated into different membranes than their native counterparts. To partially mitigate against this problem, localization tags can direct unnatural lipids to desired organelle membranes.

\section{Acknowledgments}

Work in the Baskin laboratory is supported by the NIH (R01GM131101 to J.M.B.), the Arnold and Mabel Beckman Foundation (Beckman Young Investigator, J.M.B.), the NSF (CAREER CHE-1749919 to J.M.B.; GRFP DGE-1650441 to T.W.B.), and the Alfred P. Sloan Foundation (Sloan Research Fellowship to J.M.B.).

\section{Literature}

1. Bumpus TW and Baskin JM (2018) Greasing the wheels of lipid biology with chemical tools. Trends Biochem. Sci 43, 970-983 [PubMed: 30472989]

2. Thiele $\mathrm{C}$ et al. (2012) Tracing fatty acid metabolism by click chemistry. ACS Chem. Biol 7, 2004 2011 [PubMed: 22999348]

3. Jao CY et al. (2009) Metabolic labeling and direct imaging of choline phospholipids in vivo. Proc. Natl. Acad. Sci. U. S. A 106, 15332-15337 [PubMed: 19706413]

4. Höglinger D et al. (2017) Trifunctional lipid probes for comprehensive studies of single lipid species in living cells. Proc. Natl. Acad. Sci. U.S.A 114, 1566-1571 [PubMed: 28154130]

5. Bumpus TW and Baskin JM (2017) Clickable substrate mimics enable imaging of phospholipase D activity. ACS Cent. Sci 3, 1070-1077 [PubMed: 29104923]

6. Thiele $\mathrm{C}$ et al. (2000) Cholesterol binds to synaptophysin and is required for biogenesis of synaptic vesicles. Nat. Cell Biol 2, 42-49 [PubMed: 10620806]

7. Höglinger D et al. (2015) Intracellular sphingosine releases calcium from lysosomes. Elife 4, 1-20 
8. Frank JA et al. (2016) Photoswitchable diacylglycerols enable optical control of protein kinase C. Nat. Chem. Biol 12, 755-762 [PubMed: 27454932]

9. Bumpus TW and Baskin JM (2016) A chemoenzymatic strategy for imaging cellular phosphatidic acid synthesis. Angew. Chem. Int. Ed. Engl 55, 13155-13158 [PubMed: 27633714]

10. Bumpus TW et al. (2018) Ex uno plura: differential labeling of phospholipid biosynthetic pathways with a single bioorthogonal alcohol. Biochemistry 57, 226-230 [PubMed: 29095606] 